\title{
Research on Detection Algorithm for Rail Fastener Based on Computer Vision
}

\author{
Xin Liu ${ }^{a}{ }^{*}$, Hongbin Wang ${ }^{b}$, Bin Zhou ${ }^{c}$ \\ Department of Electronic and Information Engineering Lanzhou Institute of Technology Lanzhou, \\ 730050, China \\ a fallingstar0505@163.com, b516507644@qq.com, c610073363@qq.com
}

Keywords: Computer Vision; Rail Fastener; HOG Features; Nearest Neighbor Classifier

\begin{abstract}
The traditional rail detection method cannot meet the demand of line repair, so a detection algorithm for rail fastener based on computer vision is proposed, which combines projection method and scanning of pixels in specific region to position the position of fastener, and adopts gray-scale features and HOG features to describe feature vector of fastener, then uses Chi square distance classifier to extract features. The experimental result shows the algorithm is effective and feasible to a certain extent.
\end{abstract}

\section{Introduction}

Currently Chinese rail system is developing by leaps and bounds. The detection of rail components, which is a constituent in infrastructure detection, plays an important role for guaranteeing safe railway operation. The missing of rail fasteners is one of important reasons causing rail traffic accidents, as this would expedite attrition of wheels, form various defects of rail surface, and increase the possibilities of derailing. The traditional manual detection is troublesome and time-consuming, and requires high on the testing personnel, while the nondestructive detection based on computer vision is featured with non-contacting and quick velocity, which can lower labor intensity, improve measuring efficiency and accuracy[1]. Thus, in terms of rail maintenance, the future direction is comprehensively popularizing automatic track inspection equipment.

To solve the drawbacks of existing research findings, the paper combines Halcon machine vision software to propose an algorithm that can automatically detect rail fasteners. The algorithm proposed defines the crossing position of rails and sleepers based on projection method and long straight line method, then scans pixels in this region to judge the fastener' position via statistics, combines gray scale features and HOG features to extract features of fasteners, lastly detects the fastener through the nearest neighbor classifier based on Chi square distance. The experimental result shows that the algorithm can automatically recognize fasteners, thereby providing a quick and efficient way for track inspection.

\section{Composition of Detection System}

Fig. 1 is the schematic diagram for visual rail fastener detection system. Composed of line-scan digital camera, lens, light source, laser sensor, etc.[2], the system can be installed on the bottom of track inspection car. When the track inspection car moves, the line-scan digital camera continuously obtains image for field rails and related images, which are transmitted to upper computer via communication interface to be saved and analyzed. 


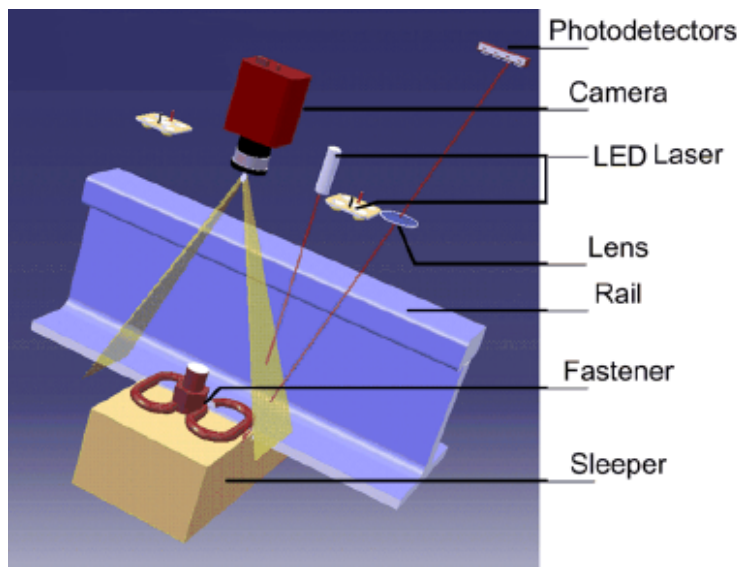

Fig. 1 The structure diagram of rail fastener detection system

The image analysis process of the system is mainly programmed using Halcon, which software is a set of machine vision integration development software developed by Germany MVtec Company and has been extensively applied to various domains of industrial production. The image processing algorithm is mainly divided into three parts: the first procedure is collecting image for preprocessing, including graying, denoising and segmenting to make later processing less complex. The second procedure is combining projection method and long straight line method to coarsely position the fastener's position. Then, the gray scale features and HOG features of rail fastener are analyzed and combined with the nearest neighbor classifier based on Chi square distance to spot the optimal fastener. Lastly the fasteners in rail picture are matched based on features to finish the detection of fastener. The whole processing flow is as shown in Fig.2.

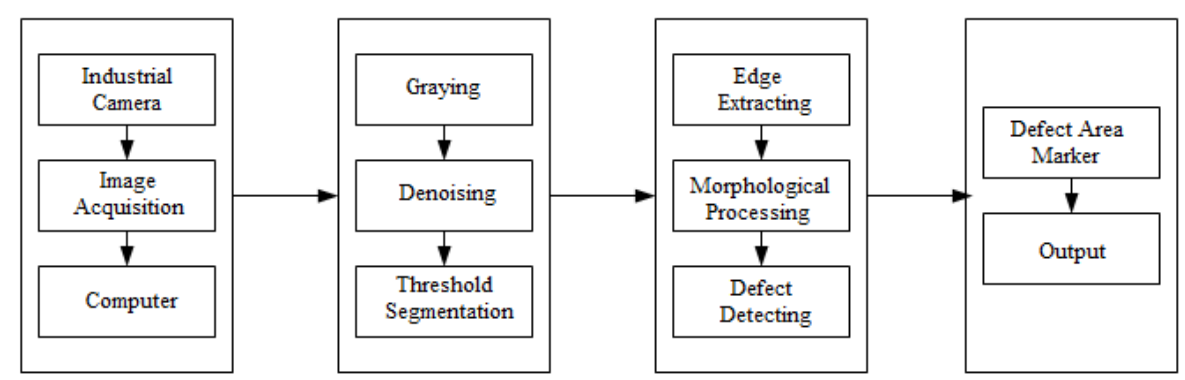

Fig.2 The flow chart of rail fastener detection system

\section{Image Preprocessing}

\subsection{Image Collection}

Image collection is mainly converting the real image into numeric picture that can be processed and stored by the computer, which process can be realized via two modes [3]: (1) reading rail fastener image from computer document via read_image operator in Halcon software; (2) connecting industrial camera via computer and adopting open_framegrabber and grab_image operators to realtimely obtain rail fastener image. In obtaining image using industrial camera, the imaging plane of camera should be as parallel as possible with the plane of measured object, or large distortion is produced, and the camera has to be calibrated. The collected rail fastener image is as shown in Fig.3. 


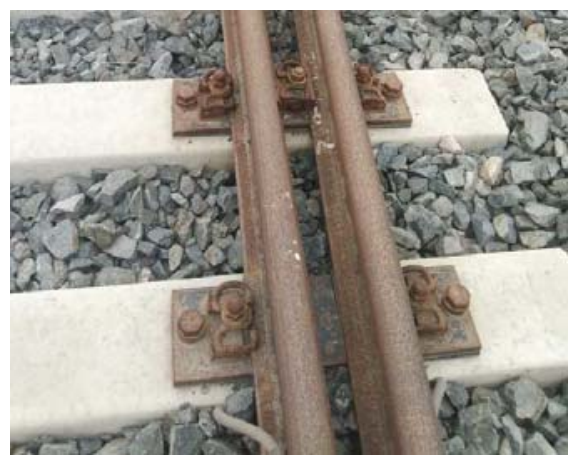

Fig.3 The image of rail fasteners

\subsection{Preprocessing}

As the resolution of linear camera used is $4096 \times 1$, and it outputs an image every 2,000 lines, the width of the collected image is greater than the region where the fastener is located, wherein the non-fastener region would influence the processing speed of processor. Besides, considering there is some noise in the image, the preprocessing includes image graying, denoising, and image segmentation.

Image graying: The images obtained via image collection are RGB color images, which need to be converted into grayscale images for subsequent image processing.

Denoising: In the process of obtaining images, the collected images all contain noise. A lot of noise in nature meets Gaussian distribution. Thus, Gaussian filtering denoising algorithm is adopted to eliminate noise. Here the paper adopts zero mean Gaussian filter operator of $3 \times 3$ [4], as shown in equation (1):

$$
G^{3}=\frac{1}{16}\left[\begin{array}{lll}
1 & 2 & 1 \\
2 & 4 & 2 \\
1 & 2 & 1
\end{array}\right]
$$

Where $G^{3}$ represents convolution template of $3 \times 3$ (the numerical value in the matrix represents filtering weight), the process of filtering is just the process in which this template is convolution with the gray scale image and then the smooth image is output.

Image segmentation: It is observed from the filtered image that the target fastener is largely different from background image in gray scale characteristics, so the binarization can be adopted for positioning, i.e. setting a threshold and the pixel whose gray scale value is smaller than the set threshold in the image is regarded as the needed target. Otherwise, the pixel belongs to background region. Here the local dynamic threshold method is mainly adopted, i.e. taking out the pixels meeting following equation (2) in each local neighborhood[5]:

$$
f_{i} \geq f_{o}+g
$$

Where $f_{i}$ is the pixel value of original gray-scale map, $f_{o}$ is pixel of filtered gray-scale map, $g$ is self-defined pixel offset. The optimal scope of pixel offset is $5 \leq g \leq 30$. Too large offset would make it difficult to extract region, while too small offset makes too many small regions with noise extracted, so the option here is $g=12$.

\section{Rail Fastener Detection}

\subsection{Coarse Positioning of Fastener Position}

As the collected images contain rail, fasteners, sleepers and other regions, and there are too many interference factors outside the target fasteners, the fasteners region needs to be positioned first before detection. In the collected image, the rails are two relatively bright and straight lines, the sleepers are perpendicular to rails, and gray scale changes in the vertical direction, while the fasteners are distributed symmetrically at the positions where rails and sleepers cross. Thus before detection of 
fasteners, the edge of rails and sleepers is firstly found out to define the approximate scope of fasteners, then the fasteners are detected within this scope. The concrete steps are as follows:

(1) Project the images in horizontal direction and vertical direction, as the region of rails is highlighted, it would show a wave crest effect in gray scale projection distribution graph, then the approximate position of rails can be detected.

(2) After projection, the longest two long line segments in this region are found out using long straight line detection method [6], and they are just the left and right boundaries of rails.

(3) With sleeper width as width of the region, tally and scan the vertical projection from left to right. As the sleepers and fasteners are significantly different, the grayed images are subjected to thresholding (here fixed threshold is selected as 80). After threading processing, the image only has tow pixel values of 0 and 1 . The number of pixel value 1 in the detected region is tallied, and the region with number of pixel value 1 greater than 9,000 is judged as sleeper region.

(4) Extract the fasteners region according to prior knowledge

Above experimental analysis shows that as the rail fasteners are always arranged on two sides of rails and the distance between fasteners is approximately $250 \sim 350$ pixels, the detected fasteners in the region can be positioned, with effect as shown in Fig.4.

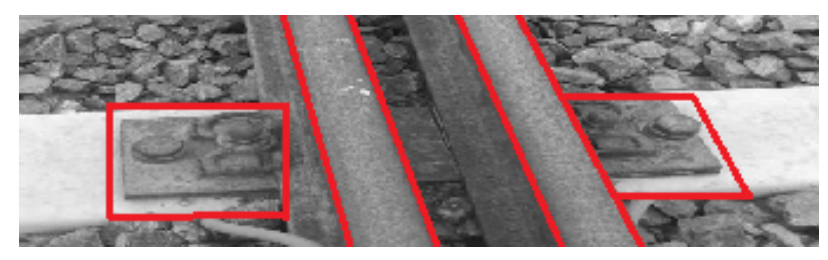

Fig.4 The result of fasteners coarse positions

\subsection{Description of Fastener's HOG Features}

There is some deviation in segmenting target fasteners only according to gray scale characteristics, so based on an analysis on gray scale characteristics, the fasteners' positions can be further defined by analyzing gradient characteristics. The HOG features can be used to obtain the shape information of fasteners via gradient and edge features of fasteners region, to provide reference for next features matching.

The process of calculating HOG features is as follows:

Calculate gradient value: The size of rail fasteners is basically $100 \times 100$, so the size of input image is $100 \times 100$, in dividing the image into cell units, the size of each cell unit is set as $100 \times 100$. Here the paper adopts one-dimensional discrete differential template to calculate the gradient of each cell unit, with calculation process[7] as shown below:

$$
\left\{\begin{array}{l}
G_{x}(x, y)=H(x+1, y)-H(x-1, y) \\
G_{y}(x, y)=H(x, y+1)-H(x, y-1)
\end{array}\right.
$$

Where $G_{x}(x, y)$ and $G_{y}(x, y)$ respectively represent the gradient of pixel $(x, y)$ in horizontal direction and vertical direction, $H(x, y)$ represents gray scale value of pixel $(x, y)$, the calculation equation for the direction of amplitude for its gradient (4) is as follows:

$$
\left\{\begin{array}{c}
G(x, y)=\sqrt{G_{x}(x, y)^{2}+G_{y}(x, y)^{2}} \\
\alpha(x, y)=\tan ^{-1}\left(\frac{G_{y}(x, y)}{G_{x}(x, y)}\right)
\end{array}\right.
$$

Where $G(x, y)$ represents the gradient magnitude of pixel $(x, y), \alpha(x, y)$ represents gradient direction of pixel $(x, y)$.

Tally the gradient direction of each pixel and conduct weighted voting for it, establish nine-dimensional (divide $0 \sim \pi$ into 9 directions) Histogram of Oriented Gradient (HOG).

Normalize the blocks and merge four neighboring cell units into a block by dividing the value on each dimension by L2 norm of vector, i.e.: 


$$
V \rightarrow \frac{V}{\sqrt{\|V\|_{2}^{2}+\xi}}
$$

Where $V$ is non-normalized vector

Combine the HOG feature vectors of all cell units in the image into a big HOG feature vector to obtain the HOG features of the whole image.

In the following, a fastener template is obtained after description of HOG features, as shown in Fig.5.

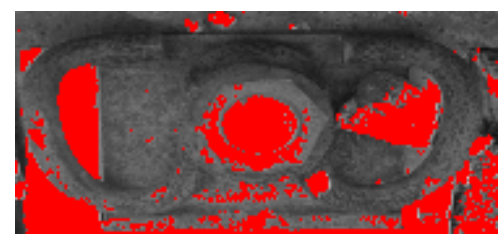

Fig.5 The fastener template

\subsection{Features Extraction of Fastener}

After the HOG features of rail fasteners are described, the feature vector of fasteners is obtained. Then the region closest to the features of fasteners template needs to be found out to finish feature extraction. The paper mainly adopts nearest-neighbor algorithm of Chi square distance as the classifier for detection of fasteners.

The main idea of nearest algorithm[8] in fastener detection is: Let sample set $D^{n}=\left\{\begin{array}{llll}x_{1} & x_{2} & \ldots & x_{n}\end{array}\right\}$, wherein, the category of each sample $x_{i}$ is known. Set the tested sample point as $x$, and set a threshold of minimum distance, the sample whose minimum distance is smaller than this threshold after compared with test sample is judged as a fastener.

Calculate Histogram distance $Y$ using Chi square distance, with concrete equation (6) as shown below:

$$
Y=\left|\frac{\mid \omega_{a}-\omega_{b}}{\omega_{a}+\omega_{b}}\right|
$$

Where ${ }^{\omega_{a}}$ is feature vector of training sample, ${ }^{\omega_{b}}$ is feature vector of tested sample

\section{Experiment Result}

The system developed is experimental system based on Halcon software. The system collected field rail video in Lanzhou western bullet train station, where the rail fasteners are spring bar I-type fasteners. 100 pictures were randomly taken for this algorithm for fasteners detection, with part of detection effect as shown in Fig.6.

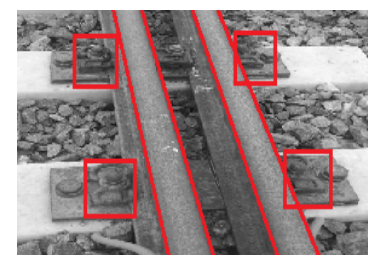

(a)

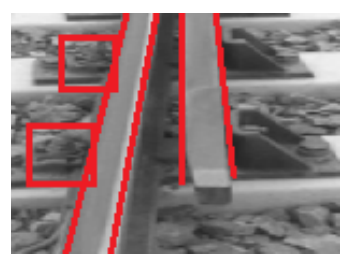

(b)

Fig.6 The results of rail fastener detection

\section{Conclusion}

The paper proposes a rail fastener detection algorithm which combines coarse positioning of fastener position and nearest-neighbor algorithm based on Chi square distance, and verifies the algorithm by collecting field video, finding that the algorithm herein can significantly detect fasteners. 
Currently the paper only experiments on the rail video collected at a slow speed, while the detection error of the algorithm would increase for the high speed video collected by track inspection car. Besides, the long straight line detection is adopted in coarse positioning of fasteners, which delivers an obvious effect for detection of straight rail, while produces some errors in detecting curving rail, leading to deviation in positioning of fasteners and an influence on detection result. Improvement on above drawbacks will be content of future research.

\section{Acknowledgements}

Introduction on authors:

Xin Liu (1983- ), female, Lanzhou, Gansu, lecturer/master, research area:intelligent information processing and image processing; Hongbin Wang(1985-), male, Pingliang, Gansu, lecturer/master; Bin Zhou(1976-), female, BaiYing, Gansu, Associate Professor.

\section{References}

[1]. Jian Yan. The problems and their solution about rail line maintenance [J]. The China Railway, 2004(11):15-19.

[2]. Fang Wu. The algorithm research and software design of fastener missing system in all vision high-speed railway [D]. The Shanghai Jiaotong University, 2013.

[3]. Xianfeng Zhu, Hongjun Pan. The defect detection and one-dimensional dimension measurement of cartridge based on Halcon [J]. The Journal of Jilin University (Information Science Edition). 2014, 32(3):308-315.

[4]. Wencheng Wang. The design of gear defect detection system based on Halcon[J]. Mechanical Drive, 2014:60-63.

[5]. Guangchun Qian, Hui Liu, Wei Tao, etc. The fast detection method of high speed moving target based on correlation-line method [J]. The Journal of Dalian Jiaotong University, 2011, 04(2):79-82.

[6]. J. Matas, C. Galambos, J. Kittler. Robust Detection of Lines Using the Progressive Probabilistic Hough Transform. Computer Vision and Image Understanding (CVIU) 78(1): 119-137, 2000.

[7]. Shan Ma. The research on railway fastener identification [D]. The Beijing Jiaotong University, 2012.

[8]. Ling Wang, Bing Zhang, Xi'ai Cheng. The detecting system of missing nut of rail fastener based on computer vision [J]. The Computer Engineering and Design, 2011, 32(12):4147-4150. 